\title{
Probing the nature of dark matter with gravitational microlensing
}

\section{Geraint Lewis*}

Institute of Astronomy, School of Physics, University of Sydney, NSW 2006, Australia

E-mail: gfl@physics.usyd.edu.au

\begin{abstract}
It has been suggested that gravitational microlensing by a stellar population embedded within a dominant distribution of smooth dark matter can explain the anomalous flux ratios observed in several multiply imaged quasars. But how smooth does this dark matter component need to be? Using a series of numerical simulations, this question is investigated, showing that there is a critical radius at which a compact dark matter distribution is indistinguishable from smoothly distributed matter. However, this critical radius is dependent upon the macrolensing parameters and the masses of the dark matter component. The same trends are seen in high magnification simulations, representative of anomalous quasar pairs, illustrating that the role of microlensing in explaining these systems requires further investigation.
\end{abstract}

The Manchester Microlensing Conference: The 12th International Conference and ANGLES Microlensing Workshop

January 21-25, 2008

Manchester, $U K$

* Speaker. 


\section{Introduction}

While $\Lambda \mathrm{CDM}$ cosmological models accurately account for the distribution of matter on the largest scales, they also predict galactic halos should possess a myriad of sub-haloes; the observational lack of these haloes has been touted as a crisis in the theory [1]. While the first generations of stars in the early universe could have removed the gas from these first collapsed objects, leaving them dark and hence unobservable within the Galaxy [2], it was noted that the gravitational lensing by such substructure could be the cause of anomalous flux ratios observed in several multiply imaged quasars $[3,4]$.

Schechter \& Wambsganss [5] re-examined question of anomalous flux ratios, but instead of invoking large dark matter clumping they focused upon the action of gravitational microlensing by compact objects along the line of sight. They found, rather counter-intuatively, that the presence of a substantial quantity ( $>90 \%$ ) of smooth dark matter resulted in a radical modification of the magnification probability distribution for negative parity (saddle-point) quasar images, with an extensive tail for substantial demagnificiation. They concluded that the anomalous ratios, could, therefore, be due to such strong microlensing demagnification, rather than the presence of clumped mass within the halo of the lensing galaxy.

But how smooth does any potential smooth dark matter have to be? Clearly any dark matter candidate is discrete on some scale and it is important to understand what factors influence the scale at which the compact nature of dark matter can be determined. This contribution summarises results presented in Lewis \& Gil-Merino [7], showing that there exists a source radius above which the microlensing of a particular compact dark matter component is indistinguishable from smoothly distributed dark matter. This resolution scale is dependent upon the macrolensing parameters and compact dark matter mass under consideration, with future work required to fully characterise for anomalous quasar systems.

\section{Approach}

\subsection{Microlensing Simualtions}

For this study, a number of microlensing simulations were undertaken using the ray-tracing approach developed by Wambsganss [8]. For these, a binary mass function was adopted, with $50 \%$ of the mass in solar mass objects, representing the stellar component, with the remaining $50 \%$ representing a dark matter component. In a series of simulations, the dark matter component was first assumed to comprised of compact objects with a mass of $1 \mathrm{M}_{\odot}$, whereas in subsequent simulations, the mass of the individual dark matter lenses was progressively reduced to a scale of $10^{-4} \mathrm{M}_{\odot}$. In the final simulation, the dark matter was assumed to be smoothly distributed, and each simulation, displayed in Figure 1, was 12 Einstein radii on a side, assuming a matter component of $\sigma=0.2$ and shear of $\gamma=0.5$.

Clearly, as the individual masses of the dark matter microlenses is reduced there is a change in the scale of the some of the features of the caustic distribution seen in the magnification map, and by a mass of $\sim 5 \times 10^{-2} \mathrm{M}_{\odot}$ the caustics possess large scale structure from the stellar masses and smaller caustics due to the dark matter. Continuing along the progression, the scale of the dark matter caustics continues to decrease until at the mass limit of our simulations, the smaller scale 


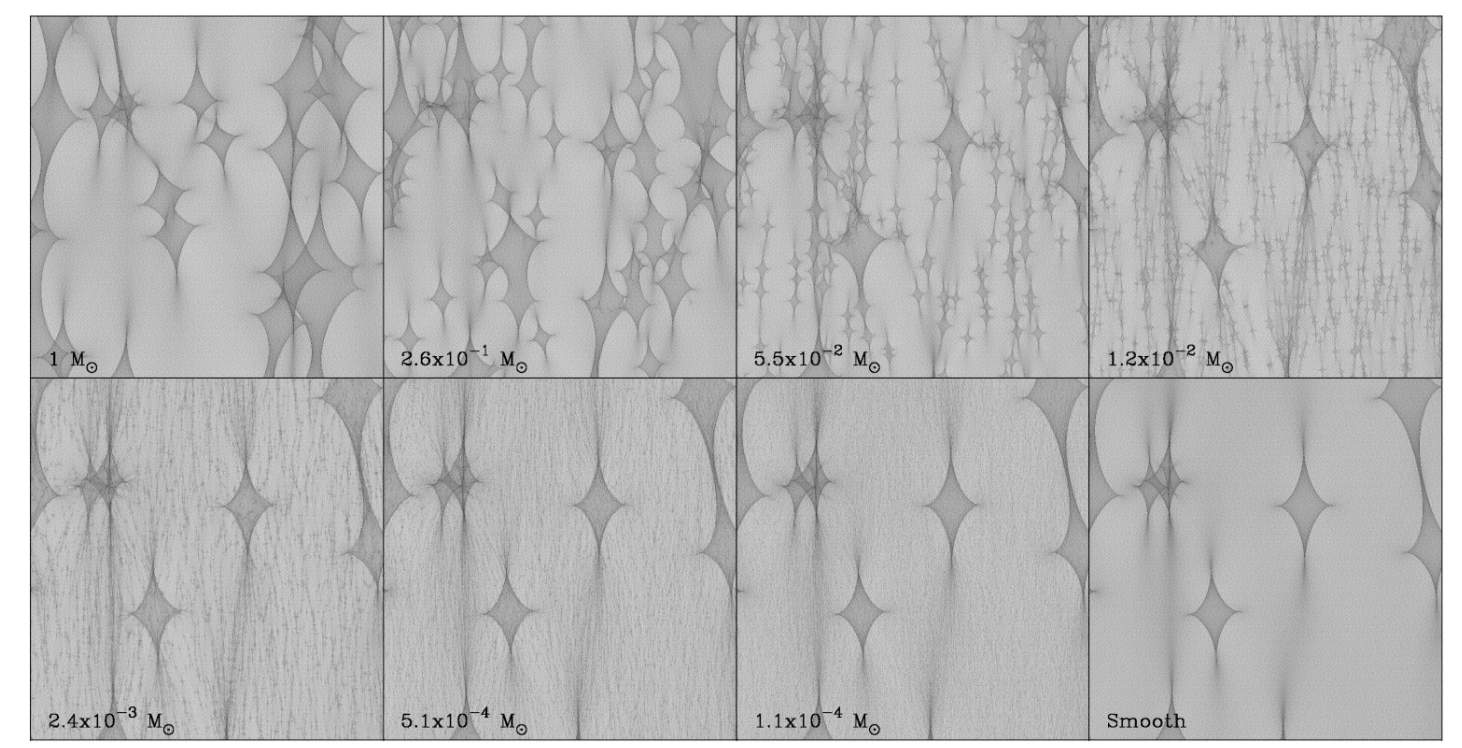

Figure 1: Magnification maps with a total surface density of $\sigma=0.2$ and shear $\gamma=0.5$. In the top-left panel, all mass is in solar mass microlenses, while in other frames $50 \%$ of the mass has been replaced by less massive microlensing masses. In the bottom-left panel, $50 \%$ of the mass component is smoothly distributed.

caustics are barely visible. If a direct comparison is made to the magnification map of the simulations with a smooth dark matter component, it is apparent that the large scale caustic structure of the two are identical, with the compact dark matter introducing a fine-scale granularity into the map; given this, it might be expected that the microlensing properties of these two maps would be very similar.

\subsection{Magnification Statistics}

Figure 2 presents an analysis of two of the microlensing simulations, the first being the case where all dark matter is distributed smoothly, whereas in the second, the dark matter is in the form of $\sim 10^{-4} \mathrm{M}_{\odot}$ compact bodies. The coloured paths across the maps result in the presented light curves, and the magnification probability distributions are presented in the right-most panel. Progressing from the top to the bottom, each map is convolved with larger sources.

In examining the top-most row, it is clear that the light curves for the smooth and compact dark matter simulations possess virtually identical large-scale variations, although the compact case possesses small-scale variations due to the granularity of the caustic map noted earlier. However, while the light curves appear to be quite similar, it is apparent that their magnification probability distributions are significantly different, with the compact masses inducing significant demagnifications that are not seen in the smooth mass case.

Progressing down the panels, it can be seen that increasing the source size blurs the magnification maps. An examination of the corresponding light curves reveals that this blurring smoothes the sharp large scale features, as expected, but the effect of smoothing is significantly more dramatic on the small scale structure present in the compact dark matter light curve; stepping through the maps this is quickly washed out and the light curves for both simulations become identical. This is 


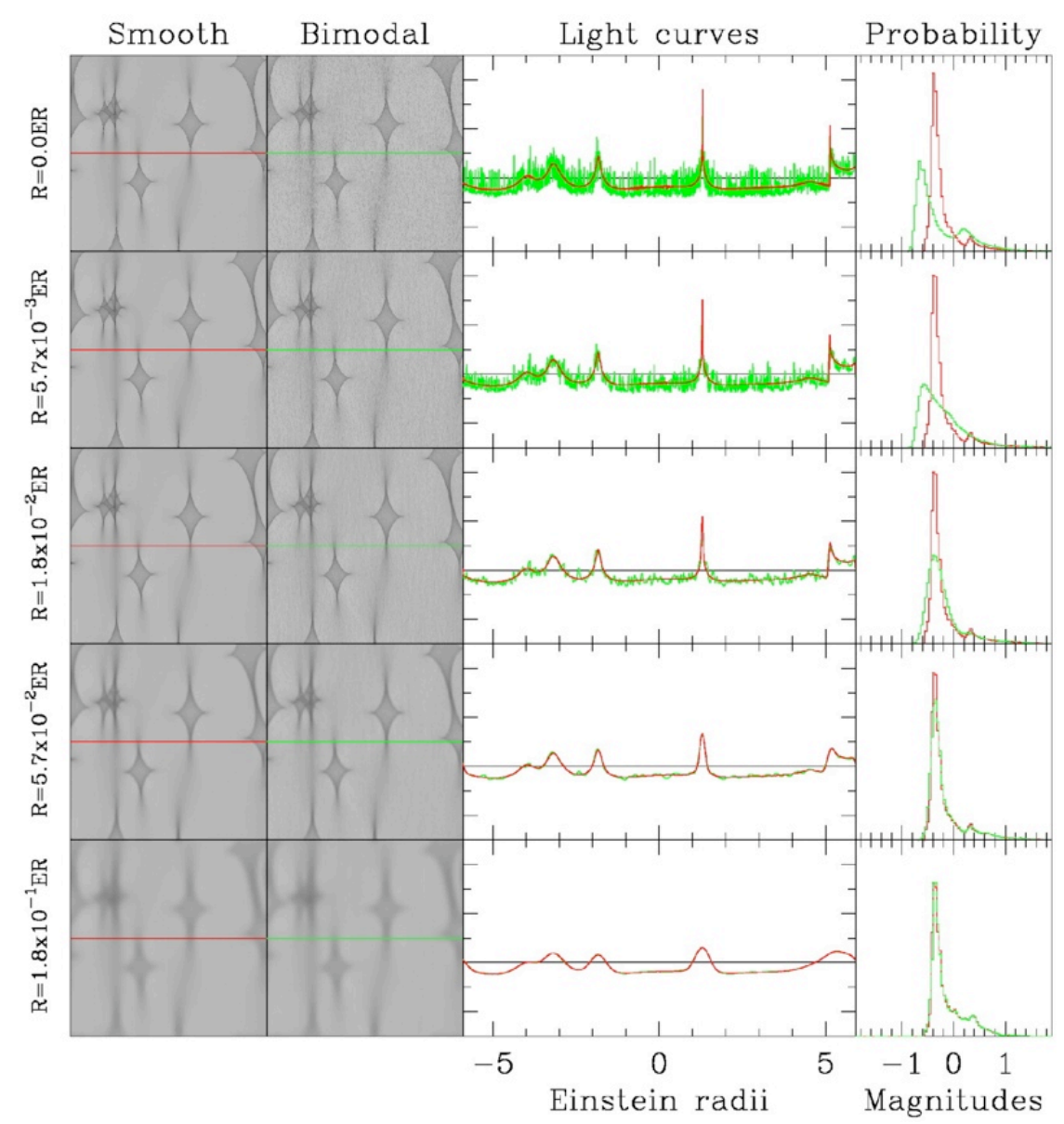

Figure 2: The two left-hand panels above presents the magnification maps for the smooth dark matter component and that with dark matter masses of $\sim 10^{-4} \mathrm{M}_{\odot}$. The light curves due to the red and green paths across these maps is presented in the next panel, whereas the final panel presents the corresponding magnification probability distributions for the two maps. Each row presents these for progressively larger source sizes.

also apparent in the magnification probability distributions, which too becomes identical when the largest source size is reached.

\subsection{Resolution Limit}

The above analysis reveals that if there is a compact dark matter component within a galaxy, then there is an effective resolution limit, set by a combination of the mass of the dark matter microlensing objects and the size of the source, below which the resulting microlensing properties are indistinguishable from a smooth matter component. To probe this resolution scale for the given suite of microlensing simulations, a simple statistical analysis was undertaken. For this, the cumulative magnification probability distribution for the smooth dark matter $\left(D_{s}\right)$ and compact dark matter $\left(D_{c}\right)$ simulations were compared, and the source size was increased until the maximum 


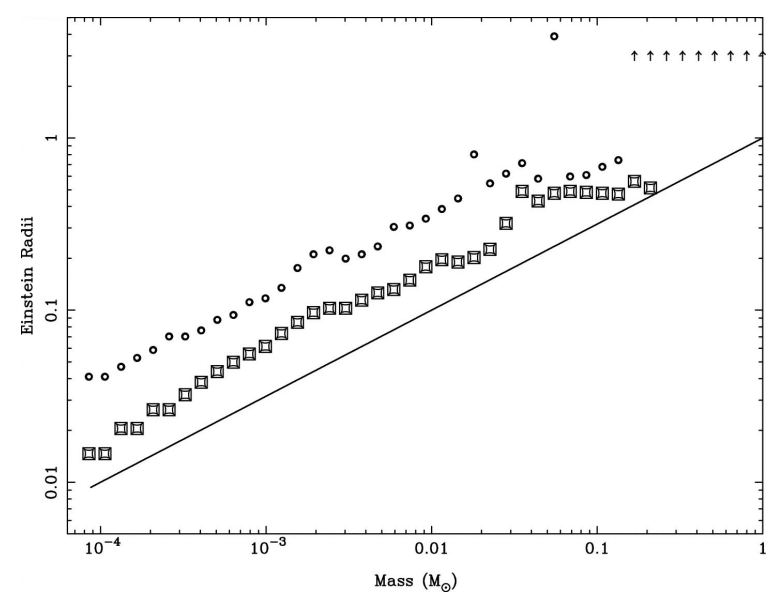

Figure 3: The limit for the difference in the cumulative magnification probability distribution to be less that $10 \%$ (squares) and 5\% (circles). The solid line in this figure corresponds to the Einstein radius associated with the dark matter masses, whereas the distances given on the abscissa is in units of the Einstein radius for a solar mass.

difference between then reached a limit, $f$, such that

$$
\operatorname{Max}\left|\frac{2 \times\left(D_{s}-D_{c}\right)}{D_{s}+D_{c}}\right|<f
$$

Figure 3 presents the results of this analysis for $f=0.05$ (circles) and $f=0.10$ (squares) for a range of compact dark matter masses. The solid line in this figure corresponds to the Einstein radius associated with the dark matter mass. As can be seen, the resolution limit scales roughly with the Einstein radius, with the $f=0.05$ limit being $\sim 3 \times E R$ for $\sim 3$ orders of magnitude in the dark matter mass; for source sizes below this limit, the dark matter will have the characteristics of compact matter, whereas above this limit, the resulting microlensing statistics will be indistinguishable from a smooth dark matter component. At large dark matter masses, however, this relation breaks down, and an examination of Figure 1 reveals that this is when the dark matter caustic structure is more than a perturbation of the caustic network induced by the stellar masses.

The above analysis was undertaken for macrolensing parameters of $\sigma=0.2$ and $\gamma=0.5$. To investigate the dependence of the resolution limit upon these parameters, three additional parameter sets were considered, with $(\sigma, \gamma)=(0.2,0.0),(0.2,0.2)$ and $(0.6,0.6)$. For each, a pair of simulations were undertaken, one with the mass made of compact dark matter with a mass of $3.5 \times 10^{-2} \mathrm{M}_{\odot}$ and the second with a dark matter mass of $1.2 \times 10^{-3} \mathrm{M}_{\odot}$, and in both cases the dark matter mass represent $50 \%$ of the total mass along the line of sight. These, again, where accompanied with simulations in which the dark matter component was smoothly distributed.

In analyzing these simulations, all where convolved with fixed source size and the resultant maps converted to magnitudes. The smooth mass magnification maps were then subtracted from the corresponding compact mass simulations, with the resulting map possessing a roughly gaussian distribution of magnitudes. Figure 4 presents the width of this gaussian distribution as a function of source radius for the above simulations; here the thin lines for the more massive dark matter component, and the thick lines for the less massive dark matter. As can be seen, for small sources, 


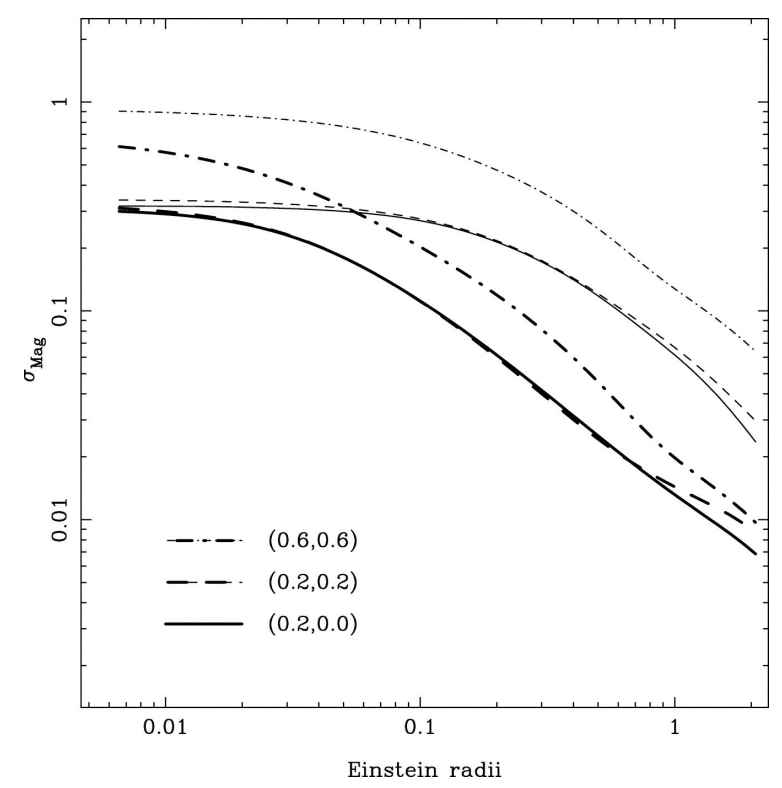

Figure 4: The difference in the magnification maps for compact and smooth dark matter simulations, as a function of the source size. The thin lines represents a compact dark matter mass of $3.5 \times 10^{-2} \mathrm{M}_{\odot}$ whereas the thick lines are for a mass of $1.2 \times 10^{-3} \mathrm{M}_{\odot}$, and the differing line styles correspond to different macrolensing parameters. Clearly, as the source size increase, the width of the distribution drops, illustrating the similarity between the compact and smooth dark matter simulations.

all cases show a a $\sigma_{M a g}$ of $0.2-1$, but as the source size increases a point is reached where this value turns over and rapidly decreases; of course, a decreasing $\sigma_{M a g}$ implies that the magnification maps are becoming similar. Hence, this turn over radius can be adopted as the critical radius at which the dark matter begins to lose its compact identity and begins to appear smooth.

\section{Anomalous Quasar Simulations}

The previous simulations, presented in [7], considered a limited range of microlensing parameters, with none similar to those that represent those for the close quasar pairs seen in anomalous systems. To this end, a new series of simulations has been undertaken considering, re-examining the models of Schechter and Wambsganss [5], although it has to be noted that the high magnifications required to explain anomalous presents significant numerical challenges due to the number of microlensing masses that are needed to represent the dark matter component.

Figures 5 and 6 presents the magnification maps for an anomalous quasar systems, with a total magnification of $\sim 10$ and $90 \%$ of the mass in a dark matter component. The upper panels presents the positive parity (time ăminimum) image, whereas the lower panels are for the negative parity (saddle point) image, with the dark matter being smoothly distributed in the left-most panel, with the other panels containing a compact dark matter component whose mass is written in the panel. Figure 5 considers a small source, whereas in Figure 6 the source size has been increased $50 \times$.

Firstly, examining the left hand panels of Figure 5, it is possible to see the deep valleys of demagnification which led Schechter and Wambsganss [5] to their conclusions. When replacing 


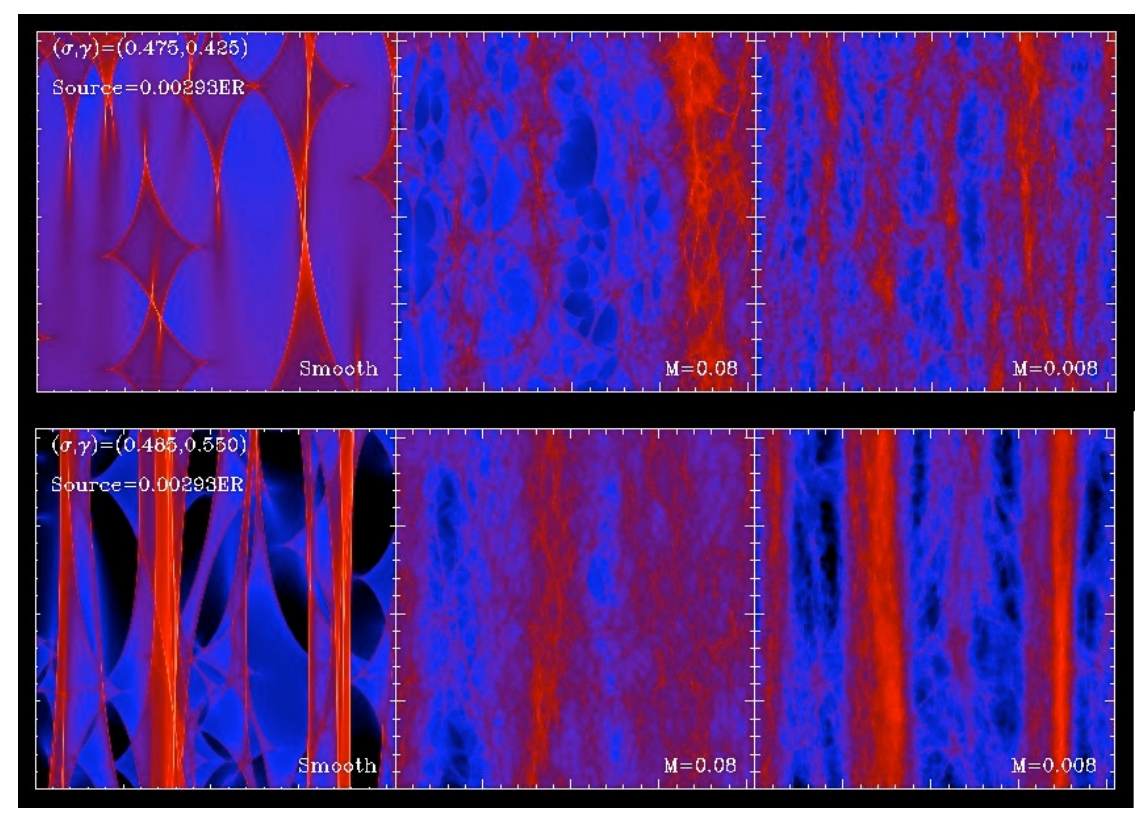

Figure 5: Magnification maps for anomalous quasar pairs, with a total magnification of $\sim 10$. The upper panels are for the positive parity images, whereas the lower panels correspond to the negative parity (saddle point) image. In these simulations, dark matter makes up $90 \%$ of the total mass, and is smoothly distributed in the left-most panels. In the remaining the panels, the dark matter comprises of compact objects with the mass written within the frame.

the smooth matter with compact matter, its clear that the resulting smaller scale caustic structure has filled in the demagnification valleys. In reducing the masses of the dark matter component further, these valleys begin to reappear and the magnification maps tend to those seen previously, with large scale caustics due to the stellar mass lenses, with a superimposed small scale structure due to the presence of the compact dark matter. In Figure 6, the larger source size again has washed out some of the caustic structure, and has filled-in the deep demagnification valleys, reducing the impact of efficacy of microlensing explaining anomalous quasars (a point noted in [9]), but a comparison of the magnification maps for the smooth matter and smallest compact matter reveals, as seen previously, the small scale caustic structure has been washed out and the maps possess very similar structures, and hence microlensing properties. Given this, we expect such systems to possess a similar critical source radius which limits the scale below which the nature of dark matter can be probed.

\section{Conclusions}

This contribution has investigated the influence of source radius on the ability to resolve whether any microlensing dark matter component is compact or smooth. It is seen that the scale is set by a number of factors, especially the masses of the dark matter component. Given this scale, however, it is clear that the microlensing of a source with a range of emission scales will display differing characteristics based upon whether the dark matter is resolved or not. Investigations of 


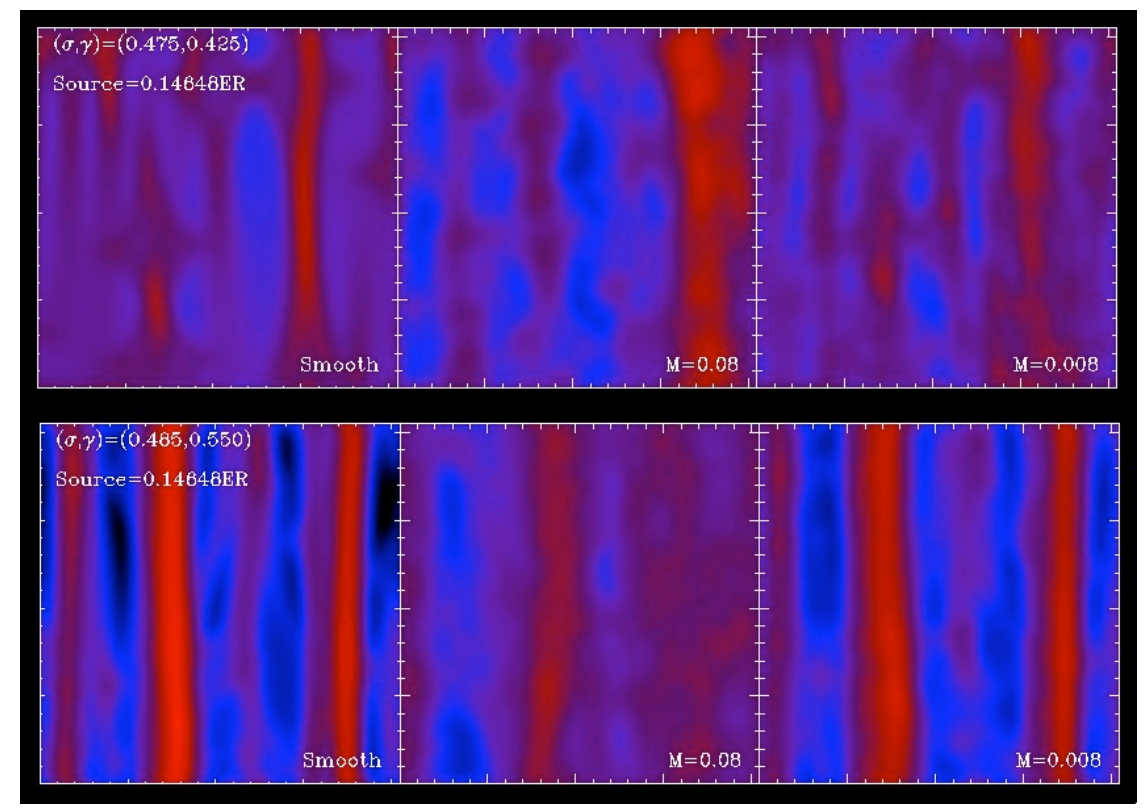

Figure 6: Same as Figure 5, for a source size of 0.15 Einstein radii.

these factors are continuing and will be presented in detail in a forthcoming article (Garsden \& Lewis, in prep.).

\section{References}

[1] A. Klypin, A. V. Kravtsov, O. Valenzuela, \& F. Prada, Where Are the Missing Galactic Satellites?, Astrophysical Journal 522 (1999) 82

[2] R. Barkana \& A. Loeb, The Photoevaporation of Dwarf Galaxies during Reionization, Astrophysical Journal 523 (1999) 54

[3] N. Dalal \& C. S. Kochanek, Direct Detection of Cold Dark Matter Substructure, Astrophysical Journal 572 (2002) 25

[4] M. Chiba, Probing Dark Matter Substructure in Lens Galaxies, Astrophysical Journal 565 (2002) 17

[5] P. L.Schechter \& J. Wambsganss, Quasar Microlensing at High Magnification and the Role of Dark Matter: Enhanced Fluctuations and Suppressed Saddle Points, Astrophysical Journal 580 (2002) 685

[6] P. L. Schechter, J. Wambsganss, \& G. F. Lewis, Qualitative Aspects of Quasar Microlensing with Two Mass Components: Magnification Patterns and Probability Distributions, Astrophysical Journal 613 (2004) 77

[7] G. F. Lewis \& R. Gil-Merino, Quasar Microlensing: When Compact Masses Mimic Smooth Matter, Astrophysical Journal 645 (2006) 835

[8] J. Wambsganss, B. Paczynski \& N. Katz, A microlensing model for QSO 2237 + 0305, Astrophysical Journal 352 (1990) 407

[9] N. F. Bate, R. L. Webster \& J. S. B. Wyithe, Smooth matter and source size in microlensing simulations of gravitationally lensed quasars, Monthly Notices of the Royal Astronomical Society $\mathbf{3 8 1}$ (2007) 1591 